\title{
Fermiology of 122 family of Fe-based superconductors: An ab initio study
}

\author{
Smritijit Sen and Haranath Ghosh \\ Indus Synchrotrons Utilization Division, Raja Ramanna Centre for Advanced Technology, Indore 452 013, India.
}

\begin{abstract}
Fermiology of various 122 systems are studied through first principles simulation. Electron doping causes expansion of electron and shrinkage of hole Fermi pockets. Isovalent Ru substitution (upto 35\%) makes no visible modification in the electron and hole like FSs providing no clue regarding the nature of charge carrier doping. However, in case of $32 \% \mathrm{P}$ doping there are considerable 'changes in the hole Fermi surfaces (FSs). From our calculations, it is very clear that two dimensionality of FSs may favour electron pair scattering between quasi-nested FSs which has important bearings in various orders (magnetic, orbital, superconducting) present in Fe-based superconductors.
\end{abstract}

Keywords: Fermi surface, Fe-based superconductors, First principles calculation

\section{Introduction}

Discovery of iron based superconductors (SCs) unwrapped a new era of superconductivity which indulges researchers to resolve the unsettled mysteries of high temperature superconductivity that remain so far unexplained within the framework of phonon mediated conventional BCS theory. Close proximity 'of superconductivity to structural and magnetic transitions, glue for the superconducting pairing, etc. are concomitant to electronic structure near Fermi energy. The Fermi surface (FS) is simply the surface in momentum space where, all the fermionic 'states with (crystal) momentum $|k|<\left|k_{F}\right|$ are occupied, and all the higher momentum states are empty. Fermi surface of Fe-based SCs mainly comprises of Fe d-orbital. Fe-based SCs have a very unusual Fermiology, which is very sensitive to doping, pressure and temperature. Angle-resolved photo-emission spectroscopy (ARPES) is one of the proficient experimental techniques by which FSs can be mapped. With the purpose of elucidating the relevance of Fermiology to the superconducting pairing mechanism from an experimental standpoint, several ARPES measurements have been performed on iron-based superconductors [1, 2, 3]. The role of FS in the development of understandings in superconductivity cannot be overemphasized [4, 5, 6, 7, 8, 9]. Electronic states of Fe-based superconductors and superconducting (SC) pairing mechanism are very much different from that of the high- $T_{c}$ cuprates [5, 10]. FS measurement is one of the probing ingredients to understand the nature of pairing of electrons in Fe-based SCs. Shape of the Fermi surface is very crucial as it determines the degree of nesting in Fe based SCs which in turn give rise to magnetic and orbital ordering [11]. Unlike spin fluctuation mediated superconductivity in cuprates and heavy fermions, orbital fluctuation has also been proposed as one of the possible pairing mechanism of superconductivity in these materials [6, 7, 12]. Orbital fluctuations are supposed to be resulting from the inter-band nesting between hole and electron like Fermi surfaces (FSs) and inter-orbital quadrupole interaction which is related to electron phonon interaction [13]. Angle-resolved photo emission spectroscopy (ARPES) measurements, have been conducted on iron-based superconductors, especially for 122 family to reveal the Fermiology of Fe-based superconductors because of availability of large numbers of high quality single crystals in this series. Theoretically calculated FS of 122 family is also available in literature [10, 14, 15, 16, 17].

The 122 family like $\mathrm{BaFe}_{2} \mathrm{As}_{2}, \mathrm{CaFe}_{2} \mathrm{As}_{2}, \mathrm{SrFe}_{2} \mathrm{As}_{2}$ etc. comprises the heart of Fe-based superconductors where best quality single crystals are available. All these materials exhibit spin density wave (SDW) order below a transition temperature and incidentally at the same temperature the structural transition from tetragonal to orthorhombic (low temperature) phase occurs. Although these parent compounds of 122 family are not superconducting but superconductivity can emerge either by applying external pressure or by chemical doping. In fact doping can be introduced at any of the sites. When $\mathrm{Ba}$ is replaced by $\mathrm{K}$ and $\mathrm{Fe}$ is replaced by $\mathrm{Co}$, it introduces hole [18] and electron doping [19] in the system respectively. The introduction of extra hole or electron shifts the chemical potential in $\mathrm{Ba}_{1-x} \mathrm{~K}_{x} \mathrm{Fe}_{2} \mathrm{As}_{2}$ [18] and $\mathrm{BaFe}_{2-x} \mathrm{Co}_{x} \mathrm{As}_{2}$ [19] in such a way that the size of the electron and hole like FSs evolve oppositely which diminishes the nesting between them, resulting suppression of spin density wave (SDW), orbital density wave (ODW) orders and SC emerges. What change in FS is then expected for isovalent substitutions? (like $\mathrm{Ru}$ substitution in $\mathrm{Fe}$ site or $\mathrm{P}$ substitution in As site) The observation of suppression of SDW order with isovalent substitution in $\mathrm{BaFe}_{2}\left(\mathrm{As}_{1-x} \mathrm{P}_{x}\right)_{2}$ and $\mathrm{BaFe}_{2-x} \mathrm{Ru}_{x} \mathrm{As}_{2}$ systems is therefore, still under debate [20, 21, 22, 23, 24]. We show that there are no significant changes either in electron or hole like FSs upon substantial $\mathrm{Ru}$ substitution and this observation is consistent with the observations of Dhaka et al., [20]. But at about 50\% doping there is significant change in the hole like FSs which is also consis- 
tent with the experimental findings by N. Xu et al.,[25]. We study on the nature of FSs when doped in any of the three sites mentioned above through first principles simulations. From our calculations we explicitly show that in case of hole doping the charge carriers go to hole FSs resulting expansion of hole FSs and shrinking of electron FSs and a reverse situation occurs in case of electron doping. In case of isovalent $\mathrm{P}$ substitution in place of As, causes substantial z-direction dispersion in the hole bands, making shape of some of the hole FSs more like three dimensional which is consistent with experiments [26, 27] and is believed to be responsible for nodal superconductivity. On the other hand, two dimensional FSs which are more favourable to nesting are believed to be responsible for high $T_{c}$ superconductivity in Fe-based materials [28]. Theoretically computed FSs of various doped 122 system are presented, similarities and dissimilarities are compared among them and with available experimental data.

\section{First principles calculation}

In our earlier work [29] a detailed structural evolution in $\mathrm{Ru}$ substituted $\mathrm{BaFe}_{2} \mathrm{As}_{2}$ pnictide superconductor as a function of $\mathrm{Ru}$ composition and temperature was carried out through combined experiment-theory study. We showed a correspondence between the anomalies in the structural parameter, particularly the displacement of the As atom and the anomalous change in resistivity across the spin density wave transition. First principles calculations to check the veracity of the structural evolution were successfully accomplished. This is significant because, one of the open problems in Fe-based materials is a difference between theoretical density functional theory (DFT) based simulations and its matching with experimental values of $z_{A s}$. Number of authors have already experimentally reported about the fact that the position of As is more closer to the Fe-plane than that observed (by more than 0.1 A) and cannot be reproduced accurately from DFT calculations [7, 29, 30, 31, 32, 33, 34]. On the other hand, various physical properties including magnetic and superconducting transitions (specially electronic structure) depend crucially on $z_{A s}$ [30]. The simulated data also explained the origin of two $\mathrm{Fe}-\mathrm{Fe}$ distances.

In a separate earlier work, we have performed a detailed electronic structure (band structure, density of states, Fermi surface structure) calculations using the experimental lattice and structural parameters $a(\mathrm{~T}, \mathrm{x}), b(\mathrm{~T}, \mathrm{x}), c(\mathrm{~T}, \mathrm{x}), z_{A s}(\mathrm{~T}, \mathrm{x})$ as input parameters in our DFT simulations that overcome above mentioned problems [30]. For clarity we explain our results of [30] briefly here. We showed that the density of states at the Fermi level for Fe-d (up as well as down spin) increases with temperature in the same fashion as that of the $z_{A s}$ i.e., due to modifications in the electronic density of states with temperature, thermal behaviour of $z_{A s}$ is dictated. The difference in up and down spin density of states at the Fermi level (that provides net magnetization) as a function of temperature shows onset of AFM like SDW state at the same temperature as that of the structural transition. Through detailed band structure calculations we showed that the $d_{x z}, d_{y z}$ bands of the Fe-d orbital be- come non-degenerate (leading to orbital ordering), both at the $\Gamma$ and $X$ points, at the structural transition temperature (above which they are degenerate). This difference in energies (of the $d_{x z}, d_{y z}$ bands) at various temperatures follows exactly the same temperature dependence of the orthorhombicity parameter determined experimentally. So far available first principles simulations on FS of Fe-based materials are based on DFT based geometrically optimized zero temperature one which do not correspond to experimental situations at room temperature. In this work, therefore, we present our simulations on FS of parent 122 and various doped 122 systems using the experimental lattice and structural parameters $a(\mathrm{~T}, \mathrm{x}), b(\mathrm{~T}, \mathrm{x}), c(\mathrm{~T}, \mathrm{x}), z_{A s}(\mathrm{~T}, \mathrm{x})$ at room temperature as fixed input parameters [19, 26, 29, 35].

Our first-principles ab-initio simulations of Fermi surfaces are performed using CASTEP module of Material studio 7.0 [36], which exploits the plane-wave pseudo-potential method based on DFT. In our simulations the electronic exchange correlation is treated under the generalized gradient approximation (GGA) using Perdew-Burke-Enzerhof (PBE) functional [37]. Geometry optimization has been carried out for two parent compounds of 122 system in which we fix $z_{A s}$. Tackling small fraction of doping $(\mathrm{Co} / \mathrm{Ru} / \mathrm{K} / \mathrm{P})$ in place of $\mathrm{Fe} / \mathrm{Ba} / \mathrm{As}$ is implemented by considering virtual crystal approximation (VCA) based on the Mixture Atom Editor of CASTEP in Material Studio 7.0. Non-spin polarized and spin polarized single point energy calculations are performed for tetragonal phase with space group symmetry I4/mmm (No.139) using ultrasoft pseudopotentials and plane wave basis set with energy cut off $500 \mathrm{eV}$ and self-consistent field (SCF) tolerance as $10^{-6} \mathrm{eV} /$ atom. Brillouin zone is sampled in the $\mathrm{k}$ space within Monkhorst-Pack scheme and grid size for SCF calculation is $25 \times 25 \times 33$. All these calculations have been carried out using primitive cell. In spin polarized calculations, spin state of the two Fe atoms have been fixed in the opposite direction so that it gives anti ferromagnetic like ordering. (a)
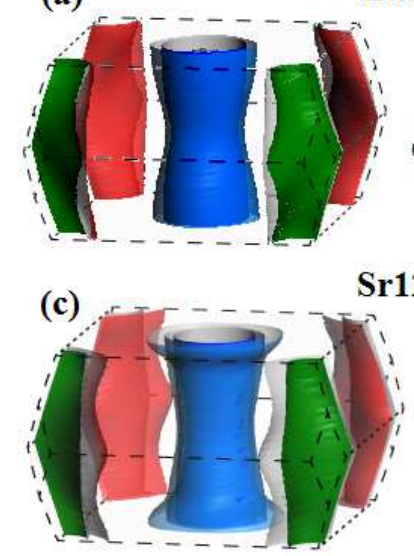

Ba122

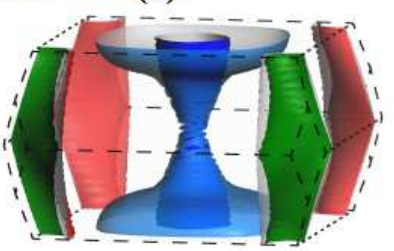

Sr122 (d)

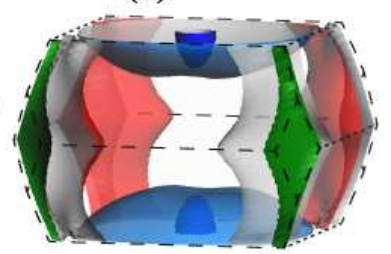

Figure 1: Calculated FSs of undoped $122 \mathrm{Fe}$-based SCs for (a) $\mathrm{BaFe}_{2} \mathrm{As}_{2}$ (b) optimized structure of $\mathrm{BaFe}_{2} \mathrm{As}_{2}$ (c) $\mathrm{SrFe}_{2} \mathrm{As}_{2}$ and (d) optimized structure of $\mathrm{SrFe}_{2} \mathrm{As}_{2}$. In each figure FSs at the center are hole like FSs, shaded blue and FSs at the corners are electron like FSs, shaded red and green. 


\section{Results and discussions}

We present simulated FS structures of 122 family of Fe-based superconductors, namely, $\mathrm{BaFe}_{2} \mathrm{As}_{2}$, $\mathrm{SrFe}_{2} \mathrm{As}_{2}, \mathrm{Ba}_{1-x} \mathrm{~K}_{x} \mathrm{Fe}_{2} \mathrm{As}_{2}, \mathrm{BaFe}_{2-x} \mathrm{Ru}_{x} \mathrm{As}_{2}, \mathrm{BaFe}_{2-x} \mathrm{Co}_{x} \mathrm{As}_{2}$, $\mathrm{BaFe}_{2} \mathrm{As}_{2-x} \mathrm{P}_{x}$. FSs have been calculated for 122 parent compounds $\mathrm{BaFe}_{2} \mathrm{As}_{2}$ and $\mathrm{SrFe}_{2} \mathrm{As}_{2}$ for unoptimized as well as optimized structures. Figure 1 depicts the simulated FSs of $\mathrm{BaFe}_{2} \mathrm{As}_{2}$ and $\mathrm{SrFe}_{2} \mathrm{As}_{2}$ where three hole like FSs appear at the centre of the brillouin zone and two electron like FSs appear at the four corners of the brillouin zone. Calculated FS topology for both parent compounds are very similar. There are experimental evidence of SDW ordering in both the parent compounds at low temperature. This SDW ordering is the result of nesting between electron and hole like FSs [11]. Two dimensional nature of the FSs in both the systems enhances nesting and results SDW ordering. Whereas FSs generated

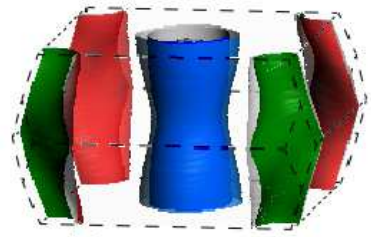

(a) Undoped

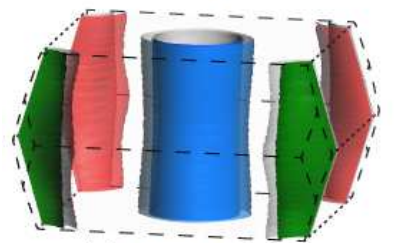

(c) $20 \% \overline{\mathrm{K}}$

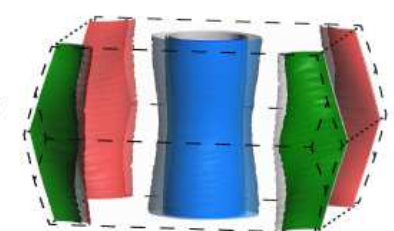

(b) $10 \% \mathrm{~K}$

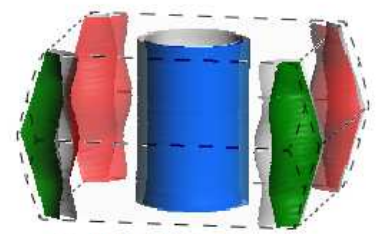

(d) $30 \% \mathrm{~K}$
Figure 2: Simulated FSs of $\mathrm{K}$ doped $\mathrm{BaFe}_{2} \mathrm{As}_{2}$ system for various $\mathrm{K}$ doping concentration

from optimized structure is more like three dimensional which works against nesting and reduces the possibility of SDW ordering. So FSs calculated using experimental lattice parameters provide more realistic FSs that resembles with the experimentally observed one [38, 39]. Even non-magnetic calculations (non spin polarized) do not make topologically appreciably different FSs. We have also studied various doped 122 systems using VCA approach. Fe d-orbital mainly constitutes the FSs of Fe based SCs. We found that at larger doping in the Fe site, VCA fails and deviates from the actual FS topology. So for some of the calculations we adopt super cell method for example $50 \%$ Ru doping. Figure 2 and Figure 3 illustrate simulated FSs of K-doped (hole doping) systems at various $\mathrm{K}$ doping concentrations. It is very clear from Figure 2 and Figure 3 that $\mathrm{K}$ doping exerts extra holes to the system which expand the hole like FSs at the centre whereas electron like FSs shrink at the corners. Exactly the reverse scenario occurs for Co doping (see Figure 4a and [5]). A more closer look on Figure 2 and Figure 3 reveals that there are significant changes in electron and hole like FSs upon $\mathrm{K}$ doping. With increasing $\mathrm{K}$ concentration the $k_{z}$ dispersion of one of the hole FS (outer one) becomes more and more weaker

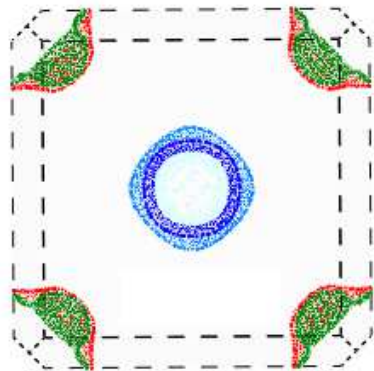

(a) Undoped

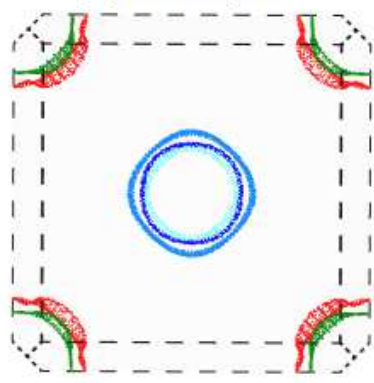

(c) $20 \% \mathrm{~K}$

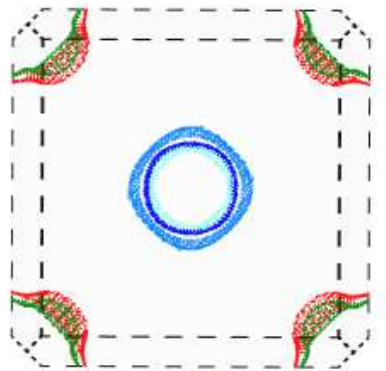

(b) $10 \% \mathrm{~K}$

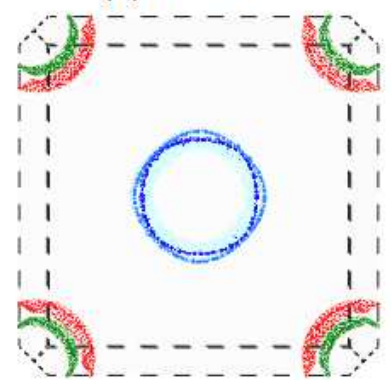

(d) $30 \% \mathrm{~K}$
Figure 3: Top view of the calculated FSs of $\mathrm{K}$ doped $\mathrm{BaFe}_{2} \mathrm{As}_{2}$ system for various $\mathrm{K}$ doping concentration (in $\mathrm{k}_{x}-\mathrm{k}_{y}$ plane) along $\mathrm{k}_{z}$ direction.

whereas around $30 \%$ doping all the hole FSs take the shape of perfect cylinder. Electron FSs also evolve significantly with doping and shows weaker $k_{z}$ dispersion with increasing doping concentration. Thus a transition in FS structure from quasi 3 dimensional to quasi 2 dimensional structure with doping is observed. As the hole and electron FSs evolve oppositely with $\mathrm{K}$ doping, the overall nesting condition degrades due to size mismatch of the FSs resulting in suppression of SDW order but at the same time two dimensionality of the FSs in these systems favours very large density of states at the Fermi level, enhancing the electron pairing possibilities. So a competing order of superconductivity and magnetism are likely to co-exist [40]. Using two band model, it was shown that apart from inter band paring, superconductivity can also arises from intra band paring and combined intra-inter band pairing results in higher $T_{c}$ compared to only inter band scenario [41]. The effect of intra band scattering also depends on the topology of FS. These facts probably explain the reason of significantly higher $T_{c}$ in $\mathrm{K}$-doped systems compared to other 122 systems. In case of Co doping with increasing doping concentration the electron FSs expand and hole FSs shrink whereas the $k_{z}$ dispersion is stronger. It is presented in figure $4 \mathrm{a}$ and figure $5 \mathrm{a}$ which is consistent with earlier theoretical and experimental results [10]. This suppresses magnetic ordering and emerges superconductivity into these systems. On the other hand, substantial $\mathrm{P}$ doping in place of As, (like 32\%) modifies hole FSs considerably as shown in figure $4 \mathrm{~b}$ and figure $5 \mathrm{~b}$. In contrast to $\mathrm{K}$ doping, P-doping in place of As, a clear dimensional cross-over from two to three dimensions is observed in the FS structure consistent with reference [42]. However, no significant changes in both the electron and hole FSs occur upon Ru substitution 


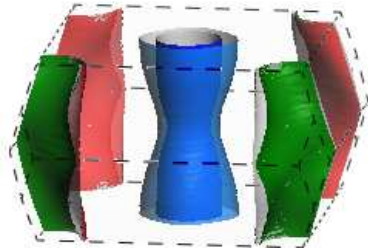

(a) $10 \% \mathrm{Co}$

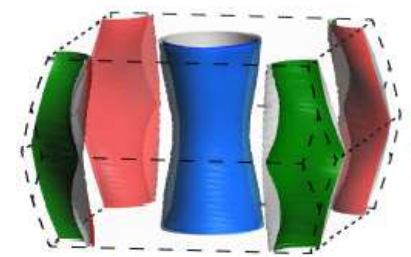

(c) $10 \% \mathrm{Ru}$

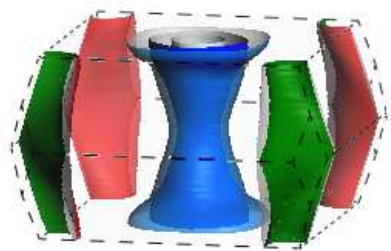

(b) $32 \% \mathrm{P}$

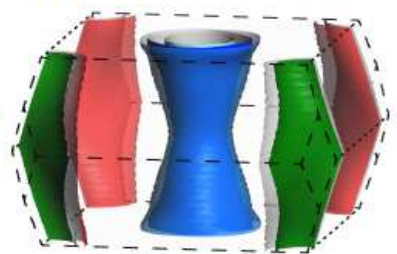

(d) $50 \% \mathrm{Ru}$

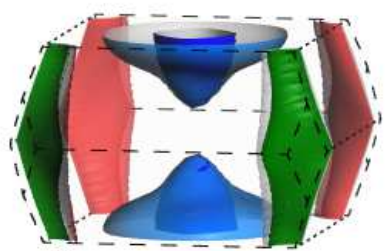

(e) $100 \% \mathrm{Ru}$

Figure 4: Fermi Surface of various doped 122 system: (a) 10\% Co doped (b) $32 \% \mathrm{P}$ doped (c) $10 \% \mathrm{Ru}$ doped(d) $50 \% \mathrm{Ru}$ doped $\mathrm{BaFe}_{2} \mathrm{As}_{2}$. (e) Calculated $\mathrm{FS}$ of $\mathrm{BaRu}_{2} \mathrm{As}_{2}$

up to $35 \%$, as was also found in experiments [20]. At about $50 \% \mathrm{Ru}$ doping there is significant modifications in the hole like FSs; more precisely, a dimensional crossover from two to three dimension occurs which is also consistent with recent experiment [25]. But calculated $\mathrm{FS}$ of $\mathrm{BaRu}_{2} \mathrm{As}_{2}$ indicates complete loss of two dimensionality of hole FSs which in turn causes large degradation of nesting. This explains the absence of magnetic order (SDW) in $\mathrm{BaRu}_{2} \mathrm{As}_{2}$ [33]. In figure 45, 4d and 4 f, evolution of FSs with Ru doping has been depicted. In figure 5a "top view" of all the FSs of figure 4 are represented to illustrate the changes in the sizes of the Fermi pockets. A closer look at all the Fermi pockets (Figure 5) reveal that there are two hole like FSs in most of the cases, one symbolized by light blue ring (outer) and other one by deep blue ring (inner). The inner and outer radii of any of the "Fermi rings" denote the radii of the FSs around $\Gamma$ and $\mathrm{Z}$ points respectively $\left(k_{z}\right.$ dispersion). These are experimentally measurable through ARPES studies and are found to be consistent with some of the observed momentum distribution curves [20]. Momentum distribution curves in general, are useful to estimate both components of the electronic self energy (real and imaginary parts) which is a powerful aspect of ARPES study. The momentum distribution curves of Fe-based materials are directly related to the Fermi surface radii because, it is a measure of electronic band structure dispersion width of a given band - how close or far (thus proportional to $\Delta k$ ) the electronic band intersects two Fermi points. In case of Co, $\mathrm{P}$ doping, changes in the sizes of the FSs and dimensionality cross-over (3D) reduce nesting and thus suppress magnetic order, e.g., $\mathrm{BaRu}_{2} \mathrm{As}_{2}$.

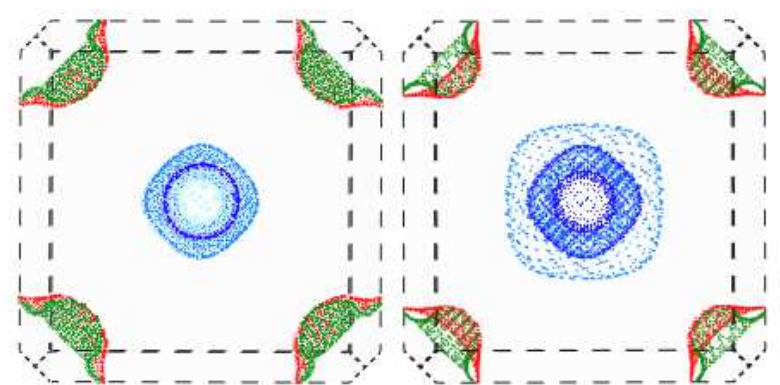

(a) $10 \%$ Co dop

(b) $32 \% \mathrm{P}$ dop

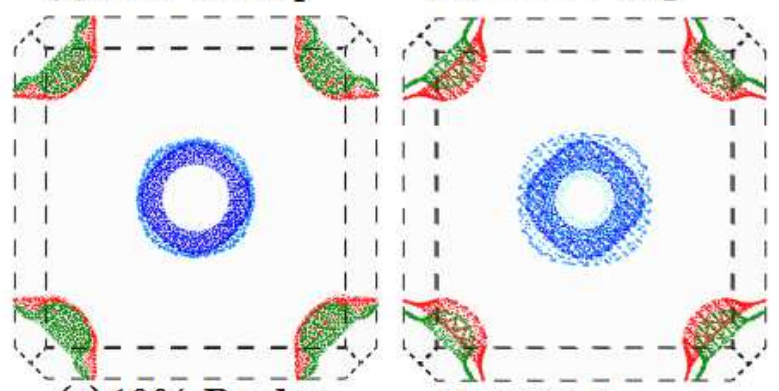

$\begin{array}{ll}\text { (c) } 10 \% & \text { Ru dop } \\ \text { (d) } 50 \%\end{array}$

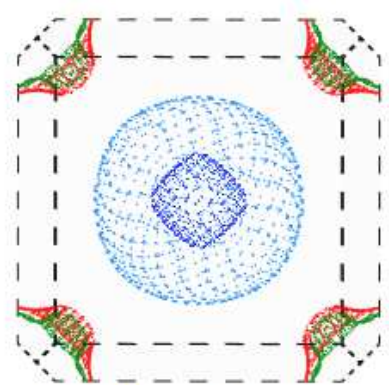

(e) $100 \% \mathrm{Ru}$

Figure 5: Top view of the FSs of various doped 122 system (in $\mathrm{k}_{x}-\mathrm{k}_{y}$ plane) along $\mathrm{k}_{z}$ direction.

All these results indicate a possible competition between superconductivity and magnetic order (SDW). SDW order (or orbital order) is controlled by inter band nesting of FSs which in turn is related to the dimensionality of the FSs. Emergence of superconductivity in these Fe-based systems seem to be originated from spin fluctuation or orbital fluctuation which may be enhanced by quasi nesting of two dimensional electron and hole FSs as suggested by M. Sunagawa et al.,[28]. Since the FSs are Fe-d orbital derived, Co-doping (which has one more d-electron than $\mathrm{Fe}$ ) clearly causes disturbance and makes modification to the electronic and hole FSs. On the other hand, $\mathrm{P}$ doping in place of As does not cause change in the d-orbital occupancy of Fe directly, but it causes changes in the pnictide height causing a z-direction dispersion. Similarly, Ru having larger atomic dimension than Fe, when replaced, the in-plane lattice parameter $a$ increases whereas the out-of-plane lattice parameter $c$ decreases after certain amount of doping, affecting $\mathrm{z}$-dispersion in band structures. Both the above two (modifications either in the pnictide height or lattice constants as described above) are not expected in case of $\mathrm{K}$ doping in place of $\mathrm{Ba}$. These are the possible origins for different 
nature of dimensional cross-over in the FS structures with substitutions in various 122 systems.

\section{Conclusions}

Calculated FSs of the parent compounds of 122 systems are very similar in topology. Presence of quasi two dimensional hole and electron like FSs enhances the chance of nesting. This is the reason why these parent compounds display magnetic and orbital ordering [28, 43]. A dimensional cross-over, in theoretically computed FS topologies of various Fe-based materials of " 122 " family are presented with various kinds of doping. Our results are consistent with experimental observations and its possible significance to magnetism and SC are presented. In case of electron and hole doping, sizes of the electron and hole Fermi pockets evolve oppositely with increasing doping concentration. Isovalent $\mathrm{Ru}$ substitution up to certain doping concentration makes no visible modifications in the electron and hole like FSs but in case of $32 \% \mathrm{P}$ doping there are substantial changes in the hole FSs. $100 \%$ Ru substitution modifies hole FSs remarkably. From all these calculated FSs it is very clear that dimensionality of FS (linked with FS nesting) plays an important role in $122 \mathrm{Fe}$-based SCs.

\section{Acknowledgement}

One of us (SS) acknowledges the HBNI, RRCAT for financial support and encouragements. We are grateful to Dr. G. S. Lodha and Dr. P. D. Gupta for their encouragements and support.

\section{References}

[1] D. H. Lu, M. Yi, S.-K. Mo, A. S. Erickson, J. Analytis, J.-H. Chu, D. J. Singh, Z. Hussain, T. H. Geballe, I. R. Fisher and Z.-X. Shen, Nature 455, 81 (2008).

[2] W. Malaeb, T. Yoshida, A. Fujimori, M. Kubota, K. Ono, K. Kihou, P. M. Shirage, H. Kito, A. Iyo, H. Eisaki, Y. Nakajima, T. Tamegai, R. Arita, J. Phys. Soc. Jpn.78, 123706 (2009).

[3] D. V. Evtushinsky, V. B. Zabolotnyy, L. Harnagea, A. N. Yaresko, S. Thirupathaiah, A. A. Kordyuk, J. Maletz, S. Aswartham, S. Wurmehl, E. Rienks, R. Follath, B. Bchner and S. V. Borisenko, Phys. Rev. B 87, 094501 (2013).

[4] G. R. Stewart, Rev. Mod. Phys. 83, 1589 (2011).

[5] Fa. Wang and D.-H. Lee, Science 332, 200 (2011).

[6] H. Kontani and S. Onari, Phys. Rev. Lett. 104, 157001 (2010).

[7] Mazin, I. I., Singh, D. J., Johannes, M. D. and Du, M. H., Phys. Rev. Lett.101, 057003 (2008).

[8] P. J. Hirschfeld, M. M. Korshunov and I. I. Mazin, Rep. Prog. Phys. 74, 124508 (2008).

[9] A. V. Chubukov, Physica C 469, 640 (2009).

[10] I. I. Mazin, and J. Schmalian, Physica C 469, 614 (2009).

[11] Haranath Ghosh and H. Purwar, Europhys. Lett. 98, 57012 (2012).

[12] K. Kuroki, S. Onari, R. Arita, H. Usui, Y. Tanaka, H. Kontani and H. Aoki, Phys. Rev. Lett.101, 087004 (2008).

[13] H. Kontani (2013), Transport Phenomena in Strongly Correlated Fermi Liquids, p.133, Heidelberg; New York: Springer, DOI 10.1007/978-3642-35365-9.

[14] I.A. Nekrasov, Z.V. Pchelkina, and M.V. Sadovskii, JETP Letters, 2008, Vol. 88, No. 2, pp. 144-149.
[15] S. Graser, A.F. Kemper, T.A. Maier, H.-P. Cheng, P.J. Hirschfeld, and D.J. Scalapino, Phys. Rev. B 81, 214503 (2010).

[16] G. Wang et al, Phys. Rev. B 81, 014521 (2010).

[17] L. Pan, J. Li, Y.-Y. Tai, M. J. Graf, J.-X. Zhu, and C. S. Ting, Phys. Rev. B 88, 214510 (2013).

[18] M.Rotter, M.Tegel, and D. Johrendt, Phys. Rev. Lett. 101, 107006 (2008).

[19] A.S. Sefat, R. Jin, M. A. McGuire, B. C. Sales, D. J. Singh and D. Mandrus, Phys. Rev. Lett.101, 117004 (2008).

[20] R. S. Dhaka, C. Liu, R. M. Fernandes, R. Jiang, C. P. Strehlow, T. Kondo, A. Thaler, J. Schmalian, S. L. Budko, P. C. Canfield and A. Kaminski, Phys. Rev. Lett.107, 267002 (2011).

[21] V. Brouet et al., Phys. Rev. Lett. 105, 087001 (2010).

[22] A. Thaler, N. Ni, A. Kracher, J.-Q. Yan, S. L. Budko, and P. C. Canfield, Phys. Rev. B82, 014534 (2010).

[23] Z. Ren, Q. Tao, S. Jiang, C.-M. Feng, C. Wang, J. H. Dai, G.-H. Cao, and Z.-A. Xu, Phys. Rev. Lett.102, 137002 (2009).

[24] Z. R. Ye et al., Phys. Rev. B 86, 035136 (2012).

[25] N. Xu, T. Qian, P. Richard, Y.-B. Shi, X.-P. Wang, P. Zhang, Y.-B. Huang, Y.-M. Xu, H. Miao, G. Xu, G.-F. Xuan, W.-H. Jiao, Z.-A. Xu, G.-H. Cao, and H. Ding, Phys. Rev. B 86, 064505 (2012).

[26] S. Kasahara, T. Shibauchi, K. Hashimoto, K. Ikada, S. Tonegawa, R. Okazaki, H. Shishido, H. Ikeda, H. Takeya, K. Hirata, T. Terashima, and Y. Matsuda, Phys. Rev. B 81, 184519 (2010).

[27] T. Yoshida, I. Nishi, S. Ideta, A. Fujimori, M. Kubota, K. Ono, S. Kasahara, T. Shibauchi, T. Terashima, Y. Matsuda, H. Ikeda, and R. Arita, Phys. Rev. Lett.106, 117001 (2011).

[28] M. Sunagawa, T. Ishiga, K. Tsubota, T. Jabuchi, J. Sonoyama, K. Iba, K. Kudo, M. Nohara, K. Ono, H. Kumigashira, T. Matsushita, M. Arita, K. Shimada, H. Namatame, M. Taniguchi, T. Wakita, Y. Muraoka and T. Yokoya, Sci. Rep. 4, 4381 (2014).

[29] S. Sharma, A. Bharathi, K. Vinod, C. S. Sundar, V. Srihari, Smritijit Sen, Haranath Ghosh, A. K. Sinha and S. K.Deb, Acta Cryst.B71, doi:10.1107/S2052520614023634. (2015).

[30] Smritijit Sen, Haranath Ghosh, A. K. Sinha and A Bharathi, Supercond. Sci. Technol. 27, 122003 (2014).

[31] D. J. Singh, Phys. Rev. B 78, 094511 (2008).

[32] I. Mazin Phys. Rev. B 78, 085104 (2008).

[33] L. Zhang and D. J. Singh, Phys. Rev. B 79, 174530 (2009).

[34] Z. P. Yin, S. Lebgue, M. J. Han, B. P. Neal, S. Y. Savrasov and W. E. Pickett, Phys. Rev. Lett.101, 047001 (2008).

[35] M. Rotter, 2010, High-Temperature Superconductivity in Doped $\mathrm{BaFe}_{2} \mathrm{As}_{2}, \mathrm{PhD}$ thesis, Ludwig-Maximilians-Universitt Mnchen, p.61.

[36] S. J. Clark, M. D. Segall, C. J. Pickard, P. J. Hasnip, M. J. Probert, K. Refson, M. C. Payne, Zeitschrift fuer Kristallographie 220, 567 (2005).

[37] J. P. Perdew, K. Burke and M. Ernzerhof, Phys. Rev. Lett. 77, 3865 (1996).

[38] H.Ding, P. Richard, K. Nakayama, K. Sugawara, T. Arakane, Y. Sekiba, A. Takayama, S. Souma, T. Sato, T. Takahashi, Z. Wang, X. Dai, Z. Fang, G. F. Chen, J. L. Luo and N. L. Wang, Europhys. Lett. 83, (2012) 47001 (2008).

[39] W.Xie, M. Bao, Z. Zhao and B.-G. Liu, Phys. Rev. B. 79, 115128 (2009).

[40] Z. Li, R. Zhou, Y. Liu, D. L. Sun, J. Yang, C. T. Lin, and G.-q. Zheng, Phys. Rev. B 86, 180501(R) (2012).

[41] Smritijit Sen and Haranath Ghosh, J. Alloy. Compd. 618, 102 (2015).

[42] S.Jiang, H. Xing, G. Xuan, C. Wang, Z. Ren, C. Feng, J. Dai, Z. Xu and G. Cao, J. Phys. Condens. Matter. 21, 382203 (2009).

[43] A. A. Kordyuk, Low Temperature Physics, 38, 9 (2012). 